\title{
The incidence of recurrent cardiovascular events among acute coronary syndrome patients treated with generic or original clopidogrel in relation to their sociodemographic and clinical characteristics. The Aegean study
}

Nikolaos G. Patsourakos ${ }^{1}$, Matina Kouvari², Apostolos Kotidis ${ }^{1}$, Kallirroi I. Kalantzi ${ }^{3}$, Maria E. Tsoumani ${ }^{3}$, Filippos Anastasiadis ${ }^{3}$, Panagiotis Andronikos ${ }^{3}$, Theano Aslanidou ${ }^{3}$, Petros Efraimidis ${ }^{3}$, Anastasios Georgiopoulos ${ }^{3}$, Kalliopi Gerakiou ${ }^{3}$, Eleni Grigoriadou-Skouta ${ }^{3}$, Panagiotis Grigoropoulos ${ }^{3}$, Dionysios Hatzopoulos ${ }^{3}$, Athanasios Kartalis ${ }^{4}$, Anastasios Lyras ${ }^{3}$, Gerasimos Markatos ${ }^{3}$, Aristeidis Mikrogeorgiou ${ }^{3}$, loannis Myroforou ${ }^{3}$, Anestis Orkopoulos ${ }^{3}$, Pavlos Pavlidis 3 , Charalampos Petras ${ }^{3}$, Maria Riga $^{3}$, Marina Skouloudi $^{3}$, Nikolaos Smyrnioudis 4 , Konstantinos Thomaidis ${ }^{3}$, Grammatiki E. Tsikouri ${ }^{3}$, Emmanuel I. Tsikouris ${ }^{5}$, Konstantinos Zisimos ${ }^{3}$, Panagiotis Vavoulis ${ }^{3}$, Maria-Gabriella Vitali ${ }^{3}$, George Vitsas ${ }^{3}$, Constantinos Vogiatzidis ${ }^{3}$, Stylianos Chantanis ${ }^{1}$, Stefanos Fousas ${ }^{1}$, Demosthenes B. Panagiotakos 2,3 , Alexandros D. Tselepis ${ }^{3}$

${ }^{1}$ Cardiology Department, Tzaneio Hospital of Piraeus, Piraeus, Greece

${ }^{2}$ School of Health Science and Education, Harokopio University of Athens, Athens, Greece

${ }^{3}$ Atherothrombosis Research Centre, University of Ioannina, Ioannina, Greece ${ }^{4}$ Cardiology Department, 'Skylitseio' General Hospital of Chios, Chios, Greece

${ }^{5}$ Cardiovascular Department, National Hospital of Kalymnos, Kalymnos, Greece

Submitted: 23 July 2019

Accepted: 9 December 2019

Arch Med Sci 2020; 16 (5): 1013-1021

DOI: https://doi.org/10.5114/aoms.2020.95878

Copyright (c 2020 Termedia \& Banach

\section{Abstract}

Introduction: The use of generic drugs is continuously growing; however, there are limited epidemiological data regarding the therapeutic equivalence of each original drug formulation with its generic counterparts. We evaluated the 12-month composite endpoint of recurrent acute myocardial infarction, ischaemic stroke, cardiac deaths, or hospitalisation due to a major bleeding in acute coronary syndrome (ACS) patients treated with original clopidogrel or a generic clopidogrel formulation, in relation to sociodemographic and clinical characteristics.

Material and methods: Consecutive Greek ACS patients $(n=1194)$ hospitalised in the Aegean islands and the Attica region were enrolled. Clopidogrel treatment was recorded either as original clopidogrel hydrogen sulphate (Plavi $\Phi^{\oplus} /$ Iscover ${ }^{\circledast}$ ) or as a generic clopidogrel besylate formulation (Clovel$\left.\mathrm{en}^{\circledast}\right)$. The composite endpoint was recorded at 12-month follow-up.

Results: The 12-month composite endpoint was 3.9\% (4.6\% in the Aegean islands and $3.5 \%$ in the Attica area, $p>0.05)$. The respective incidence in men was $4.0 \%$ and in women $3.8 \%(p>0.05)$. Overall, generic and original clopidogrel use was $87 \%$ and $13 \%$ of patients, respectively. No significant differences were observed between original and generic clopidogrel use and 12-month composite endpoint incidence. Subgroup analysis with gender, region of residence, and clinical and lifestyle factors as strata did not reveal any significant outcomes. Haemorrhage incidence did not exceed $1 \%$ in the total sample.
Corresponding author: Prof. Alexandros D. Tselepis Atherothrombosis Research Centre University of loannina loannina, Greece E-mail: atselep@uoi.gr 
Conclusions: The use of a generic clopidogrel besylate formulation was quite high in both urban and insular areas of Greece and had similar efficacy and safety profile with the original clopidogrel salt, supporting the routine use of this low-cost generic clopidogrel in the management of cardiovascular disease patients.

Key words: acute coronary syndrome, cardiovascular disease, clopidogrel besylate, gender, generic clopidogrel, secondary prevention.

\section{Introduction}

In recent years there has been a significant focus on eradicating disparities in health care due to race, sex, and region of residence throughout the world. As endorsed by the Institute of Medicine and Healthy People 2020 Agenda, the principle goal is to "improve access to comprehensive, quality health care services" for everyone [1]. Towards this perspective, successful health care systems have to maximise health gains for the entire population and simultaneously minimise the need for expensive medical care. Off-patent medicines, like generic and bioequivalent drugs, can support this objective, offering high-quality treatment at lower costs [2, 3]. Although substitution of branded medications with less expensive generic equivalents has been introduced worldwide, a high percentage of both professionals and the general public exhibit negative views on the safety and/ or effectiveness of generic drugs compared with branded ones. Acceptance of generics appears to be higher in patients with higher levels of education, while patients from lower socioeconomic demographic groups tend to have greater mistrust of these drugs [4]. In this regard, the Greek population perceives generics as being less effective and safe compared to on-/off-patent branded originator drugs, despite the fact that Greek legislation has set economic incentives in an effort to increase the use of these drugs [5]. Among this population, those with low income and young people are more likely to exhibit negative views on safety and/or effectiveness of generic drugs [6].

Cardiovascular disease (CVD) is an example of non-communicable diseases in which adequate treatment and access to a health care system is a decisive parameter in the spectrum of primary and secondary prevention, and generic drugs are increasingly investigated to be included in treatment of these patients [2]. To this issue, generic clopidogrel formulations are now used for the prevention of thrombosis in CVD patients, due to their low costs [7]. The use of these generic drugs should be widely adapted; not only in large urban centres with well-qualified health care facilities but also in remote areas with limited access to hospitals and health care services, such as islands and areas with high altitude $[8,9]$. Examining the use as well as the efficacy and safety of generic versus original clopidogrel formulations in CVD patients in these areas is of particular interest due to the different lifestyle of this population compared with urban regions in terms of less stress and higher adherence to healthy dietary patterns, i.e. Mediterranean diet [10-12].

The aim of the Aegean prospective study was to evaluate the 12-month incidence of recurrent adverse cardiovascular events and major haemorrhage in acute coronary syndrome (ACS) patients treated with original clopidogrel or a generic clopidogrel formulation in relation to their sociodemographic factors, region of residence, i.e. insular (Aegean sea islands) or urban areas of Greece (Attica region), and clinical characteristics.

\section{Material and methods}

\section{Sample}

The Aegean study is a prospective, observational cohort. During 2015-2016, a total of 1194 consecutive Greek patients with diagnosis of an ACS, i.e. acute myocardial infarction (AMI) or unstable angina (UA), who were hospitalised in the Cardiology Department of Tzaneio General-Hospital in Piraeus, Attica region and in the departments of General Hospitals in the Aegean Sea islands, were enrolled (participation rate of the initially approached ACS patients was $80 \%$ in urban area and $95 \%$ in Aegean Sea islands). The particular hospitals were selected to represent both urban areas and insular areas. Of the total enrolled patients, 740 (69\% men and 31\% women) were from urban areas and 454 from insular areas $(73 \%$ men and $27 \%$ women). Power analysis showed that the number of enrolled participants was adequate to evaluate two-sided differences between groups of the study and the investigated parameters greater than $20 \pm 5 \%$, achieving statistical power greater than 0.80 at a $5 \%$ probability level ( $p$-value).

\section{Defining ACS patients}

At hospital entry, as well as during hospitalisation, a clinical examination was performed in all patients and a complete biochemical analysis including biomarkers of cardiac injury was performed. The diagnosis of acute myocardial infarction (AMI), characterised either as ST-segment elevation (STEMI) or as high-risk unstable angina 
(UA) or AMI without ST elevation (UA/NSTEMI), was performed according to the Third Universal Definition of Myocardial Infarction [13]. ACS patients had to meet at least one of the five following diagnostic criteria: 1 . symptoms of ischaemia; 2. new (or presumably new) significant ST/T wave changes or left bundle-branch block; 3. development of pathological $\mathrm{Q}$ waves on electrocardiogram (ECG); 4. imaging evidence of new loss of viable myocardium or regional wall motion abnormality; 5. identification of intracoronary thrombus by angiography or autopsy [13]. In addition, the levels of high-sensitivity troponin I (hs-Tnl) were used as the cardiac biomarker of necrosis [13]. Medical information of the patients was retrieved through hospital records.

\section{Measurements at baseline examination}

The baseline examination included a range of patients' characteristics, selected through a case report form, and including, among others: age, gender, smoking habits, individual medical history, and family history of CVD. Specifically, years of smoking were recorded; current smokers were defined as those who smoked at least one cigarette per day or had stopped smoking during the past 12 months, while the rest were defined as past smokers; the remaining patients were defined as never or rare smokers. Regarding anthropometric characteristics, the body mass index (BMI) was calculated.

Using patients' medical files, history of stroke, heart vessel disease, atrial fibrillation, peripheral artery disease, carotid artery disease, heart failure, New York Heart Association (NYHA) functional classification, liver disease, neoplasm, and thyroid disease were recorded. Baseline assessment of other clinical characteristics (i.e. history of hypertension, hypercholesterolaemia, and diabetes mellitus) was based on the information retrieved through physical examination, as well as patients' medical records and pharmaceutical management $[12,14]$. The antiplatelet medical treatment prescribed to patients was evaluated in terms of original clopidogrel hydrogen sulphate salt (Plavix ${ }^{\circledast}$ / Iscover $^{\circledast}$ ) or generic clopidogrel besylate formulation (Clovelen ${ }^{\circledR}$ ) (600 mg loading dose, $75 \mathrm{mg}$ / day), administered in addition to aspirin (325 mg loading dose, $100 \mathrm{mg} /$ day). The clopidogrel treatment pattern was also evaluated and defined as exclusive use of generic clopidogrel or transition from original to generic clopidogrel.

\section{Follow-up}

A 12-month follow-up was performed, and information from all patients was available (i.e. $0 \%$ loss to follow-up rate). The clinical endpoint of the present study was the composite of recurrent AMI, ischaemic stroke, cardiac deaths, or hospitalisation due to a major haemorrhage, as classified according to the International Society for Thrombosis and Haemostasis (ISTH): 1. fatal bleeding and/or 2. symptomatic bleeding in a critical area or organ, such as intracranial, intraspinal, intraocular, retroperitoneal, intraarticular or pericardial, or intramuscular with compartment syndrome; and/or 3. bleeding causing a fall in haemoglobin level of $2 \mathrm{~g} / \mathrm{dl}(1.24 \mathrm{mmol} / \mathrm{l})$ or more, or leading to transfusion of two or more units of whole blood or red cells [15]. The all-cause mortality was also recorded. The follow-up was performed by outpatient visits or by phone visits. For the participants who died during the follow-up, the information was obtained from their relatives, as well as from death certificates. As regards individuals who might have first suffered from stroke and then had an AMI, it was a-priori decided that the first outcome would be considered as the end-point, but also to record the consequent event for further testing of competing risks (however, there were no such cases in the sample).

\section{Ethical consideration}

The study protocol was submitted to the Medical Research Committees of the participating Institutions, and the project was approved. The institutional review boards of Atherothrombosis Research Centre, Tzaneio Hospital of Piraeus, Skylitseio General Hospital of Chios, and the National Hospital of Kalymnos, Greece ensured that the study was carried out in accordance with the Declaration of Helsinki (1989) of the World Medical Association and its amendments. All participants were informed about the aims and procedures of the study and signed an informed consent form.

\section{Statistical analysis}

Continuous variables are presented as mean and standard deviation (SD), whereas categorical variables are shown as absolute and relative (\%) frequencies. The association between normally distributed continuous variables (BMI, age) and patients' gender was evaluated through the Student's $t$-test for independent samples. Whether these variables were normally distributed was tested through the P-P plot and equality of variances through the Levene's test. Associations between the categorical variables and gender were tested through the $\chi^{2}$ test. To evaluate the association between antiplatelet treatment (in terms of generic vs. original clopidogrel and exclusive vs. non-exclusive use of generic clopidogrel) and 12-month clinical endpoint, logistic regression analysis was performed. Results are presented as 
N.G. Patsourakos, M. Kouvari, A. Kotidis, K.I. Kalantzi, M.E. Tsoumani, F. Anastasiadis, P. Andronikos, T. Aslanidou, P. Efraimidis,

A.Georgiopoulos, K. Gerakiou, E. Grigoriadou-Skouta, P. Grigoropoulos, D. Hatzopoulos, A. Kartalis, A. Lyras, G. Markatos, A. Mikrogeorgiou,

I. Myroforou, A. Orkopoulos, P. Pavlidis, C. Petras, M. Riga, M. Skouloudi, N. Smyrnioudis, K. Thomaidis, G.E. Tsikouri, E.I. Tsikouris,

K. Zisimos, P. Vavoulis, M.G. Vitali, G. Vitsas, C. Vogiatzidis, S. Chantanis, S. Fousas, D.B. Panagiotakos, A.D. Tselepis

odds ratios (OR) and their corresponding 95\% confidence intervals $(95 \% \mathrm{Cl})$. Time to event was also taken into account, and Cox proportional hazards models were also applied, but it was decided to keep the results from logistic regression because the models had better correct classification rates for the cases and some time-points were missing. STATA software version 14 (MP \& Associates, Sparta, Greece) was used for all statistical analyses.

\section{Results}

In Table I, baseline characteristics of the ACS patients, as well as their 12-month outcome, are presented. Among ACS patients, 386 (32\%) presented with STEMI and 808 (68\%) presented with UA/NSTEMI. The overall generic and original clopidogrel use was $87 \%$ and $13 \%$, respectively; the generic drug use in insular areas being $81 \%$,

Table I. Baseline characteristics of acute coronary syndrome patients participated in Aegean epidemiological study and 12-month clinical endpoint $(n=1194)$

\begin{tabular}{|c|c|c|c|c|c|c|}
\hline \multirow[t]{2}{*}{ Baseline characteristics } & \multicolumn{3}{|c|}{ Aegean Sea Islands $(n=454)$} & \multicolumn{3}{|c|}{ Urban areas $(n=740)$} \\
\hline & $\begin{array}{c}\text { Men } \\
(n=335)\end{array}$ & $\begin{array}{l}\text { Women } \\
(n=119)\end{array}$ & $P$-value & $\begin{array}{c}\text { Men } \\
(n=513)\end{array}$ & $\begin{array}{c}\text { Women } \\
(n=227)\end{array}$ & $P$-value \\
\hline Age [years] & $65(12)$ & $69(13)$ & 0.02 & $65(11)$ & $71(10)$ & $<0.001$ \\
\hline Body mass index $\left[\mathrm{kg} / \mathrm{m}^{2}\right]$ & $28.4(4.4)$ & $28.7(7.4)$ & 0.64 & $28.2(4.0)$ & $28.7(5.6)$ & 0.24 \\
\hline Current smoking, $n$ (\%) & $174(52)$ & $20(23)$ & $<0.001$ & $297(58)$ & $74(33)$ & $<0.001$ \\
\hline Hypercholesterolemia, $n$ (\%) & $221(66)$ & $70(83)$ & 0.49 & $425(83)$ & $177(78)$ & 0.03 \\
\hline Hypertension, $n$ (\%) & $211(63)$ & $66(78)$ & 0.59 & $384(75)$ & $186(82)$ & 0.03 \\
\hline Diabetes mellitus, $n(\%)$ & $103(31)$ & $33(39)$ & 0.67 & $169(33)$ & $86(38)$ & 0.15 \\
\hline Acute myocardial infarction, $n(\%)$ & $187(56)$ & $42(50)$ & 0.01 & $261(51)$ & $93(41)$ & 0.01 \\
\hline Family history of CVD, $n$ (\%) & $60(18)$ & $8(9)$ & 0.009 & $138(27)$ & $45(20)$ & 0.04 \\
\hline Heart Failure, $n(\%)$ & $60(18)$ & $13(15)$ & 0.13 & $128(25)$ & $49(22)$ & 0.33 \\
\hline \multicolumn{7}{|l|}{ NYHA class, $n(\%):$} \\
\hline Class I & $271(81)$ & $103(87)$ & 0.35 & $384(75)$ & $179(79)$ & 0.50 \\
\hline Class II & $13(4)$ & $3(4)$ & 0.35 & $41(8)$ & $6(10)$ & 0.50 \\
\hline Class III & $29(8)$ & $3(4)$ & 0.35 & $61(12)$ & $27(9)$ & 0.50 \\
\hline Class IV & $23(7)$ & $7(5)$ & 0.35 & $25(5)$ & $3(2)$ & 0.50 \\
\hline Peripheral artery disease, $n(\%)$ & $60(18)$ & $27(32)$ & 0.03 & $61(12)$ & $13(29)$ & 0.67 \\
\hline Carotid artery disease, $n$ (\%) & $37(11)$ & $15(17)$ & 0.19 & $82(16)$ & $26(59)$ & 0.01 \\
\hline Heart valve disease, $n(\%)$ & $43(13)$ & $9(10)$ & 0.23 & $30(6)$ & $14(31)$ & 0.001 \\
\hline Atrial fibrillation, $n(\%)$ & $27(8)$ & $11(13)$ & 0.32 & $30(6)$ & $8(18)$ & 0.28 \\
\hline \multicolumn{7}{|l|}{ Antiplatelet treatment status, $n(\%):$} \\
\hline Exclusive use of original clopidogrel & $54(16)$ & $15(12)$ & 0.002 & $46(9)$ & $25(11)$ & 0.60 \\
\hline Total use of generic clopidogrel & $281(84)$ & $104(88)$ & 0.007 & $467(91)$ & $202(90)$ & 0.85 \\
\hline Exclusive use of generic clopidogrel & $197(59)$ & $62(73)$ & 0.61 & $296(67)$ & $131(65)$ & 0.03 \\
\hline Transition from original to generic clopidogrel & $84(25)$ & $42(27)$ & 0.61 & $171(33)$ & $71(35)$ & 0.03 \\
\hline \multicolumn{7}{|l|}{ 12-month follow-up: } \\
\hline Composite CVD endpoint, $n(\%)$ & $17(5.0)$ & $4(4.2)$ & 0.78 & $20(4.3)$ & $6(3.2)$ & 0.67 \\
\hline Recurrent AMI, $n(\%)$ & $10(2.7)$ & $3(2.2)$ & 0.39 & $6(1.5)$ & $0(0)$ & 0.10 \\
\hline Ischaemic stroke, $n(\%)$ & $1(0.3)$ & $0(0)$ & 0.55 & $4(0.8)$ & $1(0.6)$ & 0.88 \\
\hline Cardiac deaths, $n$ (\%) & $3(1)$ & $0(0)$ & 0.30 & $5(1)$ & $3(1.3)$ & 0.47 \\
\hline Hospitalisation due to a major haemorrhage, $n(\%)$ & $3(1)$ & $1(1)$ & 0.75 & $5(1)$ & $2(1)$ & 0.90 \\
\hline All-cause mortality, $n(\%)$ & $6(1.9)$ & $1(1)$ & 0.36 & $10(2)$ & $5(2.2)$ & 0.37 \\
\hline
\end{tabular}

Data are presented as mean (standard deviation, SD). P-values were obtained using the Student's t-test for age and body mass index and the $\chi^{2}$ test for the rest (categorical) of the variables. CVD - cardiovascular disease, NYHA - New York Heart Association Functional Classification. 
while in urban being significantly higher, i.e. $90 \%$ $(p<0.001)$.

At 12 months of follow-up the clinical endpoint (the composite of recurrent AMI, ischaemic stroke, cardiac deaths, or hospitalisation due to a major haemorrhage) had occurred in 47 patients out of 1194 (3.9\%) (4.6\% in Aegean islands and 3.5\% in urban areas, $p=0.34)$. No difference in the clinical endpoint was observed between genders (4.0\% in men and $3.8 \%$ in women patients; $p=0.86$ ). The distribution of the endpoint was AMI $40 \%$, followed by cardiac deaths $23 \%$, ischaemic stroke events $13 \%$, and the remaining $24 \%$ were re-hospitalisations due to major bleeding. Although no differences were observed between regions in the composite clinical endpoint, as regards recurrent $\mathrm{AMI}$ events, the 12-month incidence rate was $2.5 \%$ in Aegean islands versus $0.8 \%$ in urban areas $(p=0.003)$. As for all-cause mortality, no sig- nificant difference was observed between the two regions $(p>0.05)$ (Table $\mathrm{I}$ ).

Also, no significant differences were observed between those who presented a clinical endpoint or in relation to all-cause mortality and the rest, as regards the type of clopidogrel used, i.e. original or generic (all $p>0.10$ ) (Table I).

In Table II two multi-adjusted models that were developed in order to evaluate the association between the clinical endpoint at 12-months of follow-up and the clopidogrel treatment are presented. As can be seen, the type of clopidogrel used (original versus generic form) as well as the transition from original to generic clopidogrel use, did not significantly affect the 12-month clinical outcome. Moreover, the interactions between the form of clopidogrel used and sociodemographic (i.e. gender, region of residence), clinical (i.e. BMI, discharge status and history of hypercholestero-

Table II. Results from logistic regression analysis presenting the association of antiplatelet agents therapy (generic vs. original clopidogrel) (Model 1) and the pattern of clopidogrel treatment (transition from original to generic clopidogrel vs. exclusive use of generic clopidogrel) (Model 2), with the incidence of the clinical endpoint (the composite of recurrent AMI or ischaemic stroke, cardiac deaths, or hospitalisation due to a major haemorrhage) in the 12-month follow-up period $(n=1194)$

\begin{tabular}{|c|c|c|}
\hline Model 1 & OR & $95 \% \mathrm{Cl}$ \\
\hline Antiplatelet treatment (generic vs. original clopidogrel) & 1.11 & $0.45-1.71$ \\
\hline Discharge status (acute myocardial infarction/unstable angina) & 3.07 & $2.30-4.03$ \\
\hline Age (per 1 year) & 1.04 & $1.01-1.07$ \\
\hline Female gender & 0.80 & $0.40-1.60$ \\
\hline Current smoking $(y / n)$ & 1.19 & $0.62-2.30$ \\
\hline Region of residence (Aegean vs. urban) & 1.30 & $0.70-2.39$ \\
\hline Body mass index (per $1 \mathrm{~kg} / \mathrm{m}^{2}$ ) & 1.01 & $0.97-1.05$ \\
\hline Hypertension $(\mathrm{y} / \mathrm{n})$ & 0.94 & $0.46-1.89$ \\
\hline Hypercholesterolaemia (y/n) & 0.79 & $0.40-1.54$ \\
\hline Diabetes mellitus $(\mathrm{y} / \mathrm{n})$ & 1.58 & $0.85-2.92$ \\
\hline Family CVD history (y/n) & 0.86 & $0.36-2.05$ \\
\hline Model 2 & OR & $95 \% \mathrm{Cl}$ \\
\hline Transition from original to generic clopidogrel, $\mathrm{y} / \mathrm{n}$ & 1.13 & $0.62-2.06$ \\
\hline Discharge status (acute myocardial infarction/unstable angina) & 3.02 & $1.55-3.90$ \\
\hline Age (per 1 year) & 1.04 & $1.01-1.07$ \\
\hline Female gender & 0.82 & $0.41-1.65$ \\
\hline Current smoking $(\mathrm{y} / \mathrm{n})$ & 1.09 & $0.56-2.12$ \\
\hline Region of residence (Aegean vs. urban) & 1.15 & $0.61-2.14$ \\
\hline Body mass index (per 1 kg/m²) & 1.01 & $0.96-1.05$ \\
\hline Hypertension $(y / n)$ & 0.93 & $0.46-1.90$ \\
\hline Hypercholesterolaemia (y/n) & 0.76 & $0.39-1.49$ \\
\hline Diabetes mellitus (y/n) & 1.45 & $0.78-2.70$ \\
\hline Family CVD history, (y/n) & 0.67 & $0.28-1.63$ \\
\hline
\end{tabular}

OR - odds ratio, 95\% Cl - 95\% confidence interval, CVD - cardiovascular disease. 
N.G. Patsourakos, M. Kouvari, A. Kotidis, K.I. Kalantzi, M.E. Tsoumani, F. Anastasiadis, P. Andronikos, T. Aslanidou, P. Efraimidis,

A.Georgiopoulos, K. Gerakiou, E. Grigoriadou-Skouta, P. Grigoropoulos, D. Hatzopoulos, A. Kartalis, A. Lyras, G. Markatos, A. Mikrogeorgiou,

I. Myroforou, A. Orkopoulos, P. Pavlidis, C. Petras, M. Riga, M. Skouloudi, N. Smyrnioudis, K. Thomaidis, G.E. Tsikouri, E.I. Tsikouris,

K. Zisimos, P. Vavoulis, M.G. Vitali, G. Vitsas, C. Vogiatzidis, S. Chantanis, S. Fousas, D.B. Panagiotakos, A.D. Tselepis

laemia, hypertension, diabetes mellitus), and lifestyle (smoking status) characteristics on the clinical outcome, were evaluated. Stratified analyses were also performed by the aforementioned factors. As can be seen in Figures 1 and 2, the type of clopidogrel (i.e. generic vs. original) as well as the potential transition from the original to generic formulation did not significantly affect the 12-month clinical endpoint in all stratified analy- ses. This sensitivity analysis suggests that the use of either original or generic form of clopidogrel has similar effects on the 12-month clinical outcome in ACS patients, irrespective to their age, gender, region of residence, or their CVD risk factor profile.

\section{Discussion}

The 12 months following an ACS event are of crucial importance for these patients. In this work

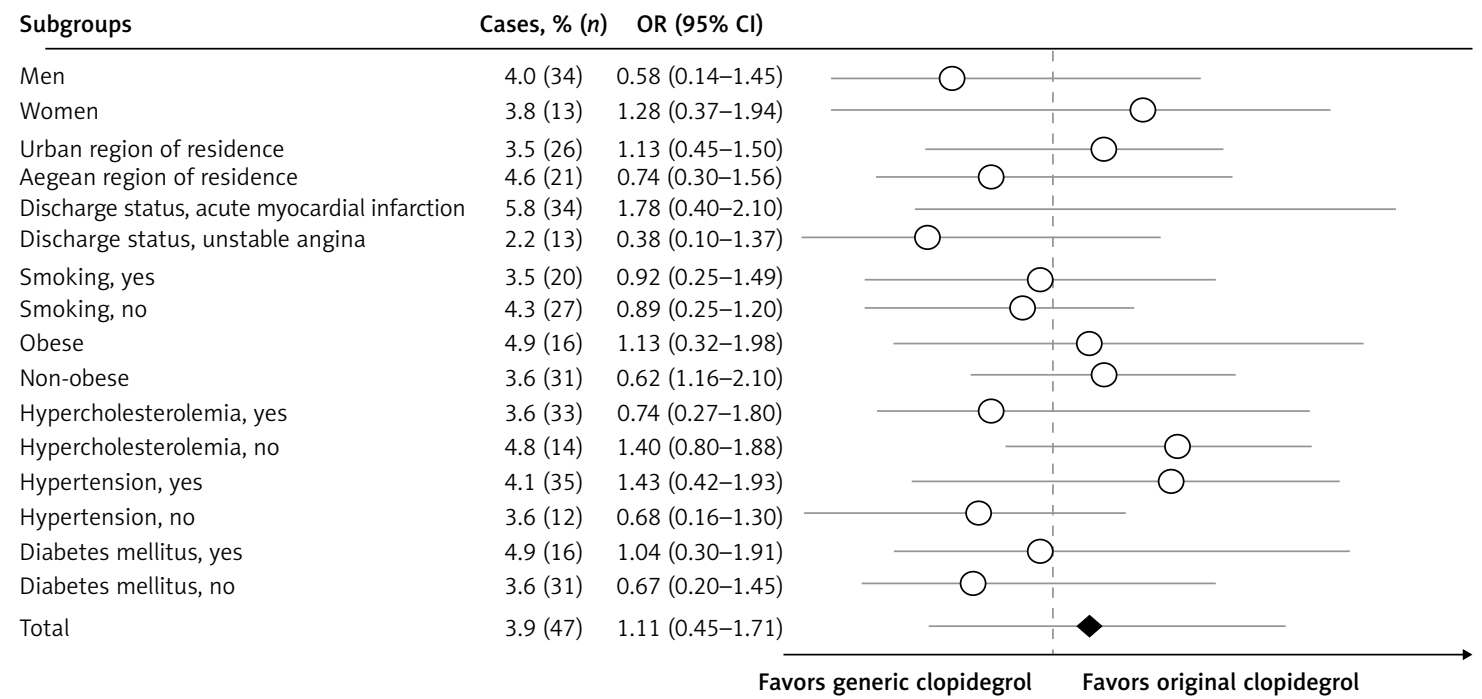

Figure 1. Stratified analysis evaluating the association between the clopidogrel formulation (generic vs. original) and 12 -month clinical composite endpoint. Odds ratios (dots) and their $95 \%$ confidence intervals (horizontal lines) were obtained through multivariate logistic regression analysis adjusted for age, sex, body mass index, region of residence, discharge status, history of hypertension, hypercholesterolemia, diabetes mellitus and family CVD history. Vertical and horizontal axes are intersected in the value 1; values in the right side indicate a favorable effect of original clopidogrel while values in the left side indicate a favorable effect of generic clopidogrel OR - odds ratio, $95 \% \mathrm{Cl}$ - 95\% confidence interval, CVD - cardiovascular disease.

\begin{tabular}{lcc} 
Subgroups & Cases, \% $(n)$ OR $(95 \% \mathrm{Cl})$ \\
\hline Men & $4.0(34)$ & $1.09(0.54-1.30)$ \\
Women & $3.8(13)$ & $1.25(0.36-1.63)$ \\
Urban region of residence & $3.5(26)$ & $1.29(0.44-1.80)$ \\
Aegean region of residence & $4.6(21)$ & $1.04(0.50-2.16)$ \\
Discharge status, acute myocardial infarction & $5.8(34)$ & $1.32(0.40-1.73)$ \\
Discharge status, unstable angina & $2.2(13)$ & $1.18(0.68-2.41)$ \\
Smoking, yes & $3.5(20)$ & $1.36(0.53-1.80)$ \\
Smoking, no & $4.3(27)$ & $0.96(0.43-2.16)$ \\
Obese & $4.9(16)$ & $0.97(0.33-1.84)$ \\
Non-obese & $3.6(31)$ & $1.18(0.56-1.50)$ \\
Hypercholesterolemia, yes & $3.6(33)$ & $1.25(0.61-1.56)$ \\
Hypercholesterolemia, no & $4.8(14)$ & $1.01(0.31-1.29)$ \\
Hypertension, yes & $4.1(35)$ & $1.16(0.58-2.32)$ \\
Hypertension, no & $3.6(12)$ & $0.95(0.27-1.36)$ \\
Diabetes mellitus, yes & $4.9(16)$ & $1.13(0.50-2.10)$ \\
Diabetes mellitus, no & $3.6(31)$ & $1.14(0.45-1.89)$ \\
Total & $3.9(47)$ & $1.13(0.62-2.06)$ \\
\end{tabular}

Figure 2. Stratified analysis evaluating the association between the exclusive use of generic versus transition from original to generic clopidogrel and 12-month clinical composite endpoint. Odds ratios (dots) and their $95 \%$ confidence intervals (horizontal lines) were obtained through multivariate logistic regression analysis adjusted for age, sex, body mass index, region of residence, discharge status, history of hypertension, hypercholesterolemia, diabetes mellitus and family CVD history. Vertical and horizontal axes are intersected in the value 1; values in the right side indicate a harmful effect while values in the left side indicate a protective effect of the tested variable OR - odds ratio, $95 \% \mathrm{Cl}$ - 95\% confidence interval, CVD - cardiovascular disease. 
it was revealed that at 12 months of follow-up, the clinical endpoint had occurred in a relatively low number of patients, below $5 \%$ of the total number of ACS patients who participated in the study, without any differences between genders, or between those patients living in mainland urban areas or on the islands. As for the all-cause mortality rate, this was low, probably due to the short-term follow-up period, the result of which was in accordance with our previously published study using the same generic clopidogrel formulation [16]. Furthermore, the use of the generic clopidogrel besylate was quite high (81-90\%) in both urban and insular areas. Despite the limitations due to the observational nature of the study, this work highlights the increasing use of this low-cost generic clopidogrel in the management of CVD patients. However, it should be stated that the use of generic clopidogrel, even in overall high levels, was lower in insular areas compared with the urban centres. The expected lower educational level of the islanders compared to the residents in urban centres may mediate this difference [4].

A number of studies aiming to compare the efficacy and safety of generic versus original clopidogrel in CVD, including ACS patients, have shown conflicting results, with some studies reporting no differences and others suggesting important potential differences [16-20]. A very recent study assessed primary causative adverse events after brand clopidogrel and generic clopidogrel use in the Food and Drug Administration (FDA) Adverse Event Reporting System. The authors demonstrated that death reports were higher, whereas the rates of cardiac, haemorrhagic, and skin complications were less common for original clopidogrel compared with generic clopidogrel formulations [21]. Therefore, it is important that any efficacy or safety concern arising from the use of generic clopidogrel in CVD patients should be referred to the specific product used. In this regard, our previous studies have demonstrated that the pharmacodynamic potency as well as clinical efficacy and safety profile of the generic clopidogrel used in the present study are similar to those of the original drug [16, 22-24]. In the present work, the 12-month composite clinical endpoint remained quite low, with the use of generic clopidogrel being at a high level, while multivariate analysis revealed that original and generic forms of clopidogrel did not present any significant differences in terms of the examined efficacy outcome (Table II). At the same time, the bleeding events remained low, probably suggesting safety equivalence as well. The present study also demonstrated that the generic clopidogrel formulation is equally effective in different subgroups, including different genders, within the studied population. Stratified analyses with gender as strata are important to confirm the drug efficacy against major events as well as its safety [25]. In the present work, both the statistical (in terms of $p$ for interaction) and the clinical (in terms of stratified risk analysis) gender-treatment interaction were evaluated, but no significant differences were observed (Figures 1 and 2). Antiplatelet treatment with clopidogrel has been investigated separately in men and women; non-significant outcomes were revealed in a meta-analysis of five clinical trials with more than 79,000 participants, $30 \%$ of whom were women [26]. However, in these meta-analysed data men presented almost twice as high recurrent CVD risk reduction compared to women (i.e. $16 \%$ vs. $7 \%$ ), while women exhibited higher bleeding risk (43\% vs. $22 \%$ ) [26]. In the previous literature, $60 \%$ of all patients admitted to the hospital for adverse drug effects are women, which can be predominantly attributed to dose-related reasons [27].

Living in remote areas of a country has long been investigated in relation to the prevalence and incidence of cardiometabolic risk factors and CVDs, mainly due to poorer health care services resulting in limited accessibility and/or delayed arrival at hospital $[8,28]$. In addition, it is expected that in these areas the target attainment in important risk factors, such as low-density lipoprotein cholesterol (LDL-C) and hypertension, the combination of which significantly increase the CVD risk, may be suboptimal as it being reported to occur even in the urban areas [29, 30]. Despite that, the present study demonstrated that patients in Aegean islands presented slightly lower, not statistically significant, hypercholesterolaemia, hypertension, and diabetes mellitus prevalence, and they were less likely to smoke (Table I). This cardiometabolic profile with lower risk factor burden could be attributed to the healthier lifestyle habits [31]. Nonetheless, the overall discrepancies in terms of clinical factors were not so evident, depicting that the current dietary habits of Mediterranean islands are not a reflection of the past; shifts towards unhealthier habits have been recorded [10]. In the present work the clinical endpoint at 12 months of follow-up was evaluated in ACS patients with clinical and demographic characteristics as strata, in relation to the type of clopidogrel used, without revealing significant differences.

All patients were treated with clopidogrel instead of ticagrelor or prasugrel as the ESC guidelines recommend for ACS patients [32]. This is primarily due to the severe economic problems during the enrolment period, which had as a consequence the prescription of a lower cost antiplatelet, compared with ticagrelor or prasugrel, i.e. clopidogrel. This led to better compliance of the 
patients participating in the present study. The findings of the present work should be considered with caution and without any causal interpretations, due to the observational nature of the study from which these results were extracted.

The aforementioned limitations are compensated by several strengths, which should be stated. Firstly, this is one of the very few studies to examine the role of generic vs. original antiplatelet treatment in CVD recurrence under the context of a "real-world" (i.e. epidemiological) study, in contrast with the strictly controlled environment of clinical trials. Secondly, a strength of the present work is related with the provision of sensitivity analysis evaluating the principal research hypothesis according to participants' gender, age, area of residence, and other factors. Lastly, to the best of our knowledge the transition from original to generic antiplatelet treatment and its association with CVD recurrence is an inadequately discussed issue in the previous literature.

In conclusion, the use of generic clopidogrel besylate was quite high in both urban and insular areas of Greece and had similar efficacy and safety profile as the original clopidogrel salt, supporting the routine use of this low-cost generic clopidogrel in the management of CVD patients.

\section{Conflict of interest}

This study was partially supported by grants from ELPEN (Greece), manufacturer of the clopidogrel besylate generic formulation (Clovelen ${ }^{\circledR}$ ) used in the present study. However, the study was investigator initiated, and the sponsor had no involvement in the design and conduct of the study, in the collection, management, analysis, and interpretation of the data or in the preparation, review or approval of the manuscript. The corresponding author had full access to all the data in the study and had the final responsibility for the decision to submit for publication. The authors have indicated that they have no other conflicts of interest regarding the content of this article.

\section{References}

1. Puerto P, Guam R, Hawaii A. Healthy people in Healthy Communities. Available from: www.healthypeople.gov.

2. Manzoli L, Flacco ME, Boccia S, et al. Generic versus brand-name drugs used in cardiovascular diseases. Eur J Epidemiol 2016; 31: 351-68.

3. Choudhry NK, Denberg TD, Qaseem A. Improving adherence to therapy and clinical outcomes while containing costs: opportunities from the greater use of generic medications: best practice advice from the Clinical Guidelines Committee of the American College of Physicians. Ann Intern Med 2016; 164: 41-9.

4. Dunne SS, Dunne CP. What do people really think of generic medicines? A systematic review and critical ap- praisal of literature on stakeholder perceptions of generic drugs. BMC Med 2015; 13: 173.

5. Vandoros S, Stargardt T. Reforms in the Greek pharmaceutical market during the financial crisis. Health Policy 2013; 109: 1-6.

6. Balasopoulos T, Charonis A, Athanasakis K, Kyriopoulos J, Pavi E. Why do generic drugs fail to achieve an adequate marketshare in Greece? Empirical findings and policy suggestions. Health Policy 2017; 121: 265-72.

7. Tsoumani ME, Kalantzi KI, Goudevenos IA, Tselepis AD. Clopidogrel generic formulations in the era of new antiplatelets: a systematic review. Curr Vasc Pharmacol 2014; 12: 766-77.

8. Mariolis A, Foscolou A, Tyrovolas S, et al. Successful aging among elders living in the mani continental region vs. insular areas of the Mediterranean: the MEDIS Study. Aging Dis 2016; 7: 285-94.

9. Panagiotakos D, Tyrovolas S, Chalkias C, et al. Health care access and prevalence of the metabolic syndrome among elders living in high-altitude areas of the mediterranean islands: the MEDIS study. Rev Diabet Stud 2011; 8: 468-76.

10. Tourlouki E, Matalas AL, Bountziouka V, et al. Are current dietary habits in Mediterranean islands a reflection of the past? Results from the MEDIS Study. Ecol Food Nutr 2013; 52: 371-86.

11. Panagiotakos DB, Notara V, Kouvari M, Pitsavos C. The Mediterranean and other dietary patterns in secondary cardiovascular disease prevention: a review. Curr Vasc Pharmacol 2016; 14: 442-51.

12. Kouvari M, Chrysohoou C, Aggelopoulos P, et al. Mediterranean diet and prognosis of first-diagnosed Acute Coronary Syndrome patients according to heart failure phenotype: Hellenic Heart Failure Study. Eur J Clin Nutr 2017; 71: 1321-8.

13. Thygesen K, Alpert JS, Jaffe AS, Simoons ML, Chaitman $B R$, White HD.; the Writing Group on behalf of the Joint ESC/ACCF/AHA/WHF Task Force for the Universal Definition of Myocardial Infarction. Third Universal Definition of Myocardial Infarction. Eur Heart J 2012; 33: 2551-67.

14. Expert Panel on Detection E and T of HBC in A. Executive Summary of the Third Report of the National Cholesterol Education Program (NCEP) Expert Panel on Detection, Evaluation, and Treatment of High Blood Cholesterol in Adults (Adult Treatment Panel III). JAMA 2001; 285: 2486-97.

15. Schulman S, Kearon C. Definition of major bleeding in clinical investigations of antihemostatic medicinal products in non-surgical patients. Subcommittee on control of anticoagulation of the Scientific and Standardization Committee of the International Society of Thrombosis an. J Thromb Haemost 2005; 3: 692-4.

16. Ntalas IV, Kalantzi KI, Tsoumani ME, et al. salts of clopidogrel: investigation to ensure clinical equivalence: a 12-month randomized clinical trial. J Cardiovasc Pharmacol Ther 2016; 21: 516-25.

17. Marcucci R, Paniccia R, Gori AM, Gensini GF, Abbate R. Bioequivalence in the real world is a complex challenge: the case of clopidogrel. J Am Coll Cardiol 2013; 61: 594-5.

18. Ashraf T, Ahmed M, Talpur MS, et al. Competency profile of locally manufactured clopidogrel Lowplat and foreign manufactured clopidogrel Plavix in patients of suspected ischemic heart disease (CLAP-IHD). J Pak Med Assoc 2005; 55: 443-8.

19. Park JB, Koo BK, Choi WG, et al. Comparison of antiplatelet efficacy and tolerability of clopidogrel napadisilate with clopidogrel bisulfate in coronary artery disease pa- 
tients after percutaneous coronary intervention: a prospective, multicenter, randomized, open-label, phase IV, noninferiority trial. Clin Ther 2013; 35: 28-37e4.

20. Ko DT, Krumholz HM, Tu JV, et al. Clinical outcomes of plavix and generic clopidogrel for patients hospitalized with an acute coronary syndrome. Circ Cardiovasc Qual Outcomes 2018; 11: e004194.

21. Serebruany VL, Hall TS, Atar D, et al. Mortality and adverse events with brand and generic clopidogrel in the US Food and Drug Administration Adverse Event Reporting System. Eur Heart J Cardiovasc Pharmacother 2019; 5: 210-5.

22. Tsoumani ME, Kalantzi KI, Dimitriou AA, Ntalas IV, Goudevenos IA, Tselepis AD. Antiplatelet efficacy of long-term treatment with clopidogrel besylate in patients with a history of acute coronary syndrome: comparison with clopidogrel hydrogen sulfate. Angiology 2012; 63: 547-51.

23. Tsoumani ME, Kalantzi KI, Dimitriou AA, Ntalas IV, Goudevenos IA, Tselepis AD. Effect of clopidogrel besylate on platelet reactivity in patients with acute coronary syndromes. Comparison with clopidogrel hydrogen sulfate. Expert Opin Pharmacother 2012; 13: 149-58.

24. Ntalas IV, Kalantzi KI, Tsoumani ME, et al. Generic clopidogrel besylate in the secondary prevention of atherothrombotic events: a 6-month follow-up of a randomised clinical trial. Curr Vasc Pharmacol 2015; 13 : 809-18.

25. Kouvari M, Yannakoulia M, Souliotis K, Panagiotakos DB. Challenges in Sex- and gender-centered prevention and management of cardiovascular disease: implications of genetic, metabolic, and environmental paths. Angiology 2018; 69: 843-53.

26. Berger JS, Bhatt DL, Cannon CP, et al. The relative efficacy and safety of clopidogrel in women and men: a sex-specific collaborative meta-analysis. J Am Coll Cardiol 2009; 54: 1935-45.

27. Xhyheri B, Bugiardini R. Diagnosis and treatment of heart disease: are women different from men? Prog Cardiovasc Dis 2010; 53: 227-36.

28. Pitsavos C, Kourlaba G, Panagiotakos DB, Stefanadis C. Factors associated with delay in seeking health care for hospitalized patients with acute coronary syndromes: the GREECS study. Hellenic J Cardiol 2006; 47: 329-36.

29. Liberopoulos E, Rallidis L, Spanoudi F, et al. Attainment of cholesterol target values in Greece: results from the Dyslipidemia International Study II. Arch Med Sci 2019; 15: 821-31.

30. Dai S, Huang B, Zou Y, Liu Y. Associations of dipping and non-dipping hypertension with cardiovascular diseases in patients with dyslipidemia. Arch Med Sci 2019; 15: 337-42.

31. Tourlouki E, Polychronopoulos E, Zeimbekis A, et al. The "secrets" of the long livers in Mediterranean islands: the MEDIS study. Eur J Public Health 2010; 20: 659-64.

32. Valgimigli $M$, Bueno $H$, Byrne RA, et al.; ESC Scientific Document Group; ESC Committee for Practice Guidelines (CPG); ESC National Cardiac Societies. 2017 ESC focused update on dual antiplatelet therapy in coronary artery disease developed in collaboration with EACTS: The Task Force for dual antiplatelet therapy in coronary artery disease of the European Society of Cardiology (ESC) and of the European Association for Cardio-Thoracic Surgery (EACTS). Eur Heart J 2018; 39: 213-60. 